\title{
Balance hídrico mensual de una cuenca Patagónica de Chile: Aplicación de un modelo parsimonioso
}

\section{Monthly water balance of a Patagonia Basin of Chile: application of a parsimonious model}

Fecha de entrega: 23 de agosto 2012 Fecha de aceptación: 13 de noviembre 2012

\section{José Vargas $^{1}$, Luis De La Fuente ${ }^{2}$ y José Luis Arumí ${ }^{3}$}

\footnotetext{
${ }^{1}$ Departamento de Ingeniería Civil, Universidad de Concepción, Casilla 160-C, Correo 3, Concepción, Chile, jvargas@udec.cl

${ }^{2}$ RyQ Ingeniería S.A., Miguel Claro 578, Providencia, Santiago, Chile, luis.delafuente@ryq.cl

${ }^{3}$ Departamento de Recursos Hídricos, Universidad de Concepción, Chillán, Chile, jarumi@udec.cl
}

La necesidad de conocimiento especifico del funcionamiento e interacción de los fenómenos hidrológicos en una cuenca, hace necesario la aplicación de modelos hidricos mensuales que permitan cuantificar la variación de los almacenamientos en una zona donde los datos existentes son escasos o muy recientes. Este trabajo se realizó en el periodo hidrológico desde 1980/1981 al 2000/2001 en la cuenca del río Simpson (subcuenca del río Aysén), ubicada en la Región de Aysén, Patagonia

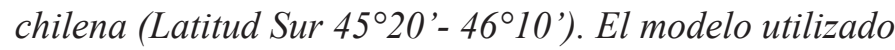
corresponde a un modelo parsimonioso de 6 parámetros ligeramente modificado a las características de la zona en estudio, para el cual se posee una distribución espacial de la precipitación y evapotranspiración potencial, obtenida con geoestadística y evapotranspiración de referencia de Hargreaves y Samani. Como resultado se obtuvo la distribución mensual de los almacenamientos con correlaciones del modelo de 0.88 en la calibración y 0.83 en la validación, y un error del balance hídrico medio anual menor al $5 \%$.

Palabras clave: balance hídrico, modelo parsimonioso, Patagonia chilena
The need for specific knowledge of the functioning and interaction of hydrological phenomena in a basin necessitates the application of monthly water models to quantify the change in storage of an area where collected data are scarce or very recent. This work was performed in the hydrologic period from 1980/1981 to 2000/2001 in the Simpson river basin (basin of the river Aysen), located in the Aysen Region, Chilean Patagonia (South Latitude $\left.45^{\circ} 20^{\prime}-46^{\circ} 10^{\prime}\right)$. A parsimonious model of 6 parameters, slightly modified to the characteristics of the study area, is used; for which a spatial distribution of precipitation and potential evapotranspiration is obtained with geostatistic and Hargreaves and Samani reference evapotranspiration. The result was a monthly distribution of the storage model with correlations of 0.88 and 0.83 for the calibration and validation periods, respectively, and an average annual water balance error less than 5\%.

Keywords: water balance, parsimonious model, Chilean Patagonia

balances, por lo cual el uso de modelos hidrológicos de paso mensual permite suplir aparentemente esta escasez de datos y avanzar en el conocimiento a la espera que en el futuro sea posible validar los resultados de estos.

\section{Descripción de la cuenca}

La cuenca hidrográfica del río Simpson, área de estudio del proyecto, se encuentra en su mayor parte en una zona climática intermedia caracterizada por la presencia 
de un clima trasandino con degeneración estepárica de

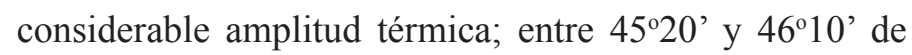
Latitud Sur y 71'25' y 72³0' de Longitud Oeste (Figura 1). La cuenca abarca una superficie de $3.975 \mathrm{~km}^{2}$ y acoge a aproximadamente 50.000 habitantes de la Región de Aysén, Chile (DGA, 2004). La topografía del sector presenta una gran variabilidad de Oeste a Este debido a la presencia de la cordillera de los Andes en la zona occidental del área de estudio con cotas de aproximadamente $2000 \mathrm{msnm}$, para llegar a la zona más oriental de la cuenca con alturas aproximadas de $700 \mathrm{msnm}$.

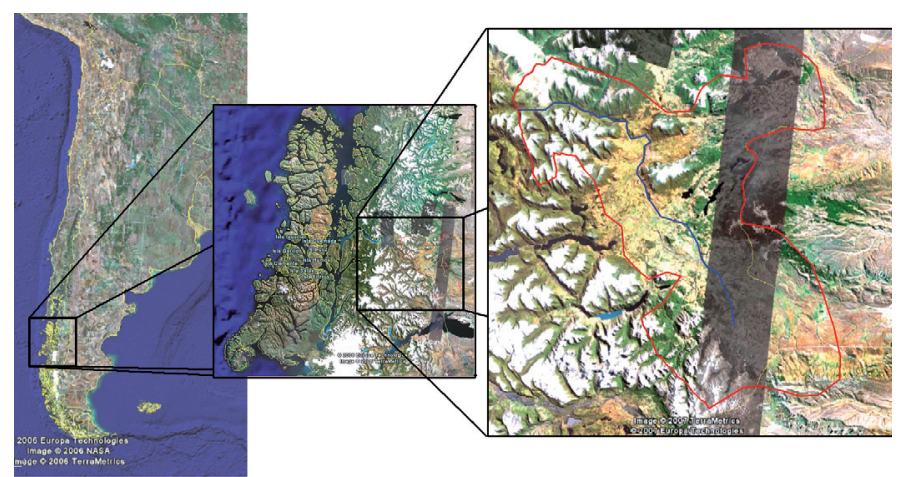

Figura 1: Ubicación geográfica de la guenca del río Simpson. Fuente: Google Earth.

\section{Balance hídrico}

La ecuación del balance hídrico (1), indica los valores relativos de entrada y salida de flujo y la variación del volumen de agua almacenada en una región. En general, las entradas en la ecuación del balance hídrico comprenden la precipitación $\mathrm{P}$, en forma de lluvia o nieve, y las aguas superficiales y subterráneas $\mathrm{Q}_{\mathrm{r}}$. Las salidas incluyen la evaporación desde la superficie de la masa de agua E, la evapotranspiración desde el suelo y la vegetación ETR, y la salida de agua superficial y subterránea desde la cuenca $\mathrm{Q}_{\mathrm{S}}$. Cuando las entradas superan a las salidas el volumen de agua almacenada $\Delta \mathrm{S}$ aumenta y cuando ocurre lo contrario disminuye.

$$
\frac{\Delta \mathrm{S}}{\Delta \mathrm{t}}=\mathrm{P}+\mathrm{Q}_{\mathrm{I}}-\mathrm{E}-\mathrm{ETR}-\mathrm{Q}_{\mathrm{S}}
$$

El almacenamiento en una cuenca $\Delta \mathrm{S}$ se puede dividir básicamente en nival $\Delta$ Snow, superficial $N$ (lagos y red de drenaje, $\Delta \mathrm{S}$ ), subsuperficial $\Delta \mathrm{Sb}$ y subterráneo $\Delta \mathrm{G}$. Considerando estos parámetros en (1) resulta:
$\frac{\Delta \text { Snow }}{\Delta \mathrm{t}}+\frac{\Delta \mathrm{N}}{\Delta \mathrm{t}}+\frac{\Delta \mathrm{Sb}}{\Delta \mathrm{t}}+\frac{\Delta \mathrm{G}}{\Delta \mathrm{t}} \approx \mathrm{P}-\mathrm{E}-\mathrm{ETR}-\mathrm{Q}_{S}$

La determinación del balance hídrico para un año medio es el caso más simple, ya que se puede despreciar la variación del volumen de agua almacenada en la cuenca $\Delta \mathrm{S}$. Para un largo período, los incrementos del agua almacenada, positivos y negativos, a escala anual, tienden a equilibrarse y su valor neto al final puede considerarse igual a cero. La situación contraria ocurre cuando se calculan los balances hídricos para períodos cortos, para los cuales $\Delta \mathrm{S} \neq 0$. Cuanto más corto es el intervalo de tiempo, más preciso debe ser la medida y determinación de los componentes del balance hídrico y más subdividido estarán los valores de $\Delta \mathrm{S}$ y de otros elementos. Esto da como resultado una ecuación del balance compleja, que es difícil de ajustar aceptablemente cuando hay escases de datos de campo (Sokolov et al., 1981).

Las medidas y determinaciones de los elementos del balance hídrico siempre tienen errores, por lo que generalmente el balance hídrico no es exacto. La inexactitud viene representada por un término residual $\eta$, que incluye los errores en la determinación de los componentes y los valores de los componentes que no se han tenido en cuenta. Con lo cual la ecuación final para cualquier $\Delta t$ es la siguiente,

$$
\frac{\Delta \text { Snow }}{\Delta \mathrm{t}}+\frac{\Delta \mathrm{N}}{\Delta \mathrm{t}}+\frac{\Delta \mathrm{Sb}}{\Delta \mathrm{t}}+\frac{\Delta \mathrm{G}}{\Delta \mathrm{t}}=\mathrm{P}-\mathrm{E}-\mathrm{ETR}-\mathrm{Q}_{\mathrm{S}}+\eta
$$

\section{Balance hídrico anual 1987}

El primer Balance Hídrico realizado en la zona fue hecho en 1987 y fue realizado por la Dirección General de Agua DGA de Chile. Las series utilizadas correspondían a datos entre 1961 y 1980. En este balance se consideró un balance hídrico medio anual con lo cual los términos de almacenamiento en (3) tienden a cero. El método para la determinación del balance hidrológico utilizó además el trazado de mapas de isoyetas e isotermas medias anuales que permitió deducir el mapa de isolíneas de evapotranspiración de superficies naturales según la expresión de Turc. 


$$
\mathrm{ET}=\frac{\mathrm{P}}{\sqrt{0.9+\left(\frac{\mathrm{P}}{\mathrm{L}}\right)^{2}}}, \text { válida si } \mathrm{P}>0.31 \mathrm{~L}
$$

Donde ET es la evapotranspiración real en mm/año, P es la precipitación media anual en mm/año y L es definido por la siguiente ecuación donde $\mathrm{T}$ es la temperatura media anual en ${ }^{\circ} \mathrm{C}$.

$$
\mathrm{L}=300+25 \mathrm{~T}+0.05 \mathrm{~T}^{3}
$$

La metodología para cerrar el balance consideró dos situaciones, las cuencas con y sin control fluviométrico. En el primer caso, al poder evaluarse en forma independiente los términos de precipitación, caudal y evapotranspiración se obtuvo un valor de discrepancia $\eta$. Si dicho término había sido demasiado grande entonces se modificaba el trazado de las isoyetas hasta encontrar un $\eta$ aceptable. En el segundo caso cuando no se disponía de control fluviométrico, el término de discrepancia $\eta$ no era posible de determinarse, por lo cual la ecuación de balance fue utilizada para deducir el valor de la escorrentía faltante. El término de discrepancia $\eta$ se consideró aceptable si era menor al $10 \%$, si no se debió iterar para conseguir un error aceptable de acuerdo a lo anteriormente mencionado. Con esta metodología se obtuvo el Balance Hídrico para la cuenca del río Simpson, el cual es resumido en Tabla 1.

Tabla 1: Balance Hídrico Anual de la Cuenca del Río Simpson (1961-1980)

\begin{tabular}{|c|c|c|c|c|c|c|c|c|}
\cline { 2 - 9 } \multicolumn{1}{c|}{} & \multicolumn{2}{c|}{ Precipitación } & \multicolumn{2}{c|}{ Escorrentía } & \multicolumn{2}{c|}{$\begin{array}{c}\text { Evapotrans- } \\
\text { piración Real }\end{array}$} & \multicolumn{2}{c|}{ Evaporación } \\
\hline $\begin{array}{c}\text { Superficie, } \\
\mathrm{km}^{2}\end{array}$ & $\begin{array}{c}\mathrm{mm} / \\
\text { año }\end{array}$ & $\mathrm{m}^{3} / \mathrm{s}$ & $\begin{array}{c}\mathrm{mm} / \\
\text { año }\end{array}$ & $\mathrm{m}^{3} / \mathrm{s}$ & $\begin{array}{c}\mathrm{mm} / \\
\text { año }\end{array}$ & $\mathrm{m}^{3} / \mathrm{s}$ & $\begin{array}{c}\mathrm{mm} / \\
\text { año }\end{array}$ & $\mathrm{m}^{3} / \mathrm{s}$ \\
\hline 3676 & 1458 & 170 & 884 & 103 & 440 & 51.3 & 2.57 & 0.3 \\
\hline
\end{tabular}

Fuente: Balance Hídrico de Chile (DGA, 1987)

La discrepancia estimada fue de $-131 \mathrm{~mm} / \mathrm{año}$, un $-9 \%$ con respecto a la variable de mayor magnitud.

\section{Descripción de componentes del balance hídrico}

En el balance hídrico de una cuenca, toda la información debe encontrarse integrada, con lo cual cada componente poseerá metodologías diferentes de integración. A continuación se realiza una descripción general de la información disponible y un resumen de la integración de cada variable.

\section{Precipitación}

La información disponible consta de 11 estaciones pluviométricas indicadas en la Tabla 2 y distribuidas como se muestra en la Figura 2. A partir de estos registros de precipitación mensual se hace una interpolación espacial con herramientas geoestadísticas obteniéndose un archivo semejante a una malla rectangular (grilla). Una vez generado este archivo se obtiene el valor en cada una de las celdas de la malla (UNESCO-IMTA, 2006).

Tabla 2: Estaciones pluviométricas disponibles

\begin{tabular}{|l|c|c|c|c|c|}
\hline \multicolumn{1}{|c|}{ Estación } & $\begin{array}{c}\text { Periodo en } \\
\text { servicio }\end{array}$ & $\begin{array}{c}\text { Latitud } \\
\circ\end{array}$ & $\begin{array}{c}\text { Longitud } \\
\circ\end{array}$ & $\begin{array}{c}\text { Altura, } \\
\text { msnm }\end{array}$ & $\begin{array}{c}\text { Precip. } \\
\text { media } \\
\text { anual, } \\
\text { mm }\end{array}$ \\
\hline Estero Perdido & $1981-1987$ & 453230 & 724000 & 80 & 3460.4 \\
\hline $\begin{array}{l}\text { Puerto } \\
\text { Chacabuco }\end{array}$ & $1965-2005$ & 452803 & 724900 & 10 & 3385.1 \\
\hline Puerto Aysén & $1931-2004$ & 452403 & 724200 & 10 & 2786.6 \\
\hline $\begin{array}{l}\text { Desagüe Lago } \\
\text { Caro }\end{array}$ & $1981-1987$ & 454745 & 723607 & 285 & 2473.5 \\
\hline El Balseo & $1980-2004$ & 452403 & 722900 & 150 & 1621.1 \\
\hline Villa Mañihuales & $1986-2004$ & 451003 & 720900 & 150 & 1603.2 \\
\hline $\begin{array}{l}\text { Coyhaique } \\
\text { Escuela Agrícola }\end{array}$ & $1984-2004$ & 453403 & 720200 & 343 & 937.5 \\
\hline Villa Ortega & $1980-2004$ & 452203 & 715901 & 550 & 766.0 \\
\hline Balmaceda & $1980-1987$ & 455500 & 714100 & 520 & 564.0 \\
\hline Nirehuao & $1993-2004$ & 451503 & 714301 & 535 & 551.4 \\
\hline Coyhaique Alto & $1962-2004$ & 452903 & 713601 & 730 & 318.8 \\
\hline
\end{tabular}

\section{Escorrentía}

La cuenca en estudio actualmente no posee una estación de cierre, con lo cual se hace necesario realizar una transposición de caudales desde estaciones insertas en la cuenca que presenten comportamiento hidrológico similar y cuyos datos estadísticos sean confiables y están indicadas en la Tabla 3. Las estaciones disponibles indicadas en la Tabla 3 se muestran en la Figura 2. 
Tabla 3: Estaciones fluviométricas disponibles

\begin{tabular}{|l|c|c|c|c|c|}
\hline \multicolumn{1}{|c|}{ Estación } & $\begin{array}{c}\text { Periodo } \\
\text { en servicio }\end{array}$ & $\begin{array}{c}\text { Latitud } \\
\circ\end{array}$ & $\begin{array}{c}\text { Longitud } \\
\text { ! " }\end{array}$ & $\begin{array}{c}\text { Área, } \\
\mathrm{km}^{2}\end{array}$ & $\begin{array}{c}\text { Caudal } \\
\text { medio } \\
\text { anual } \\
\text { específico, } \\
\mathrm{mm}\end{array}$ \\
\hline $\begin{array}{l}\text { Río Coyhaique } \\
\text { en Tejas Verdes }\end{array}$ & $1979-2003$ & 453403 & 720200 & 605.8 & 305.7 \\
\hline $\begin{array}{l}\text { Río Huemules } \\
\text { frente a cerro }\end{array}$ & $1979-2003$ & 455017 & 714637 & - & 183.9 \\
\hline $\begin{array}{l}\text { Galera } \\
\begin{array}{l}\text { Río Simpson } \\
\text { después de junta } \\
\text { con Coyhaique }\end{array}\end{array}$ & $1969-2003$ & 453303 & 720400 & 3219.2 & 406.3 \\
\hline
\end{tabular}

La estadística fluviométrica a la salida de la cuenca Q se convierte a caudal específico $\mathrm{Q}_{\mathrm{S}}$, donde $\mathrm{A}$ es área de la cuenca.

$$
\mathrm{Q}_{\mathrm{S}}=\frac{\mathrm{Q}}{\mathrm{A}}
$$

La estación base utilizada corresponde a río Coyhaique en Tejas Verdes, por ser la que presenta mayor cantidad de años completos y por ser la que entrega mayor confianza en la toma de datos luego de la visita a terreno realizada. Además, esta subcuenca posee una distribución de la vegetación similar a la cuenca completa. No obstante lo anterior, la estación base fue utilizada solo para completar y ampliar la estadística de la estación del río Simpson bajo junta Coyhaique y no para la generación de la estadística en el punto de cierre de la cuenca (río Simpson antes de la junta del río Mañihuales, sector Balseo). Esto debido a que la relación entre el área de esta subcuenca y la cuenca completa no es la adecuada, es decir, la estación base posee un área de $606 \mathrm{~km}^{2}$ versus la estación del río Simpson después de junta con Coyhaique que posee un área de $3219 \mathrm{~km}^{2}$ y la cuenca completa del río Simpson que posee $3972 \mathrm{~km}^{2}$. Posteriormente se realizó la transposición desde la estación del río Simpson hasta el punto de cierre de la cuenca.

\section{Evapotranspiración y evaporación}

Los mismos factores que dominan la evaporación de una superficie de agua abierta generan la evapotranspiración:

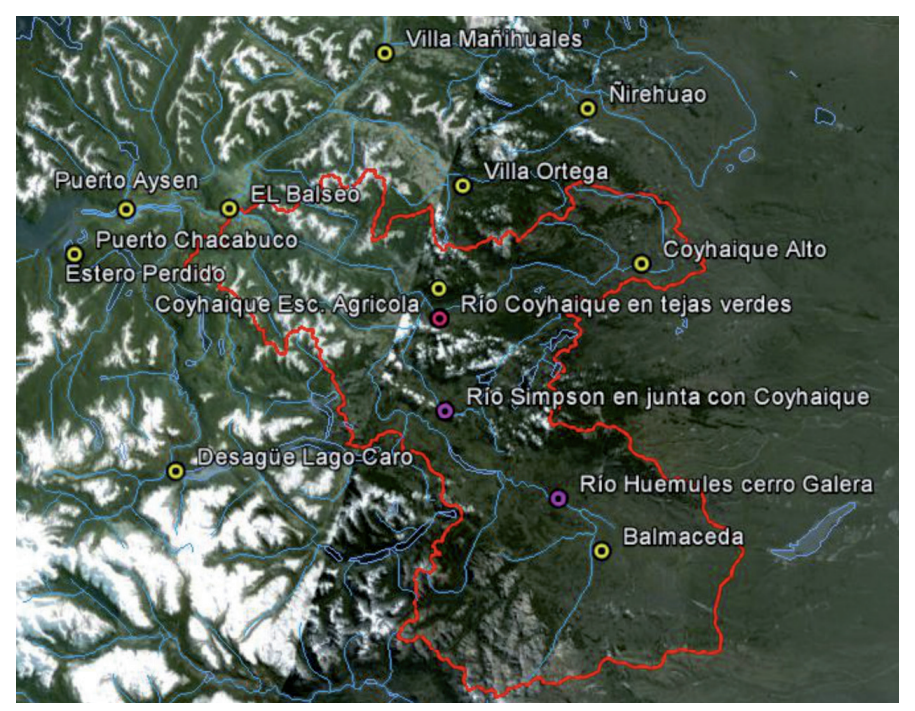

Figura 2: Distribución espacial de las estaciones pluviométricas (amarillo) y fluviométricas (morado)

suministro de energía y transporte de vapor. Para la determinación de la evapotranspiración de referencia se posee información de viento, evaporación de bandeja, horas de sol, humedad relativa, temperaturas máximas, medias y mínimas. Sin embargo, la única información que posee un grado aceptable de confianza y datos suficientes por años, son los de temperatura. Por lo cual, se utilizó el método Hargreaves (Hargreaves y Samani, 1985) para la determinación de la evapotranspiración de referencia, el cual necesita solamente datos de temperaturas y de radiación solar. El proceso siguiente fue determinar el coeficiente de cultivo, que permite convertir desde el cultivo de referencia a cualquier cobertura vegetal que exista en la cuenca. Las estaciones meteorológicas utilizadas se muestran en la Tabla 4.

Se construyeron mapas de isotermas que en conjunto con la información de uso de suelo hace posible estimar la evapotranspiración potencial. La evaporación desde superficies de agua abiertas es incorporada dentro de la evapotranspiración debido a la baja importancia relativa según su porcentaje de área $(0.7 \%)$ y según el balance hídrico publicado por DGA (1987).

\section{Almacenamientos}

Según DGA (1987) el almacenamiento puede subdividirse en: almacenamiento de agua en los suelos no saturados, de agua subterránea en los acuíferos, en lagos y embalse, en los cauces de los ríos, en glaciares y en el manto de nieve. 
Tabla 4: Estaciones térmicas utilizadas

\begin{tabular}{|c|c|c|c|c|c|}
\hline Estación & $\begin{array}{c}\text { Periodo en } \\
\text { servicio }\end{array}$ & $\begin{array}{l}\text { Lat. } \\
\circ, " ~\end{array}$ & $\begin{array}{c}\text { Long. } \\
\circ, " ~\end{array}$ & $\begin{array}{l}\text { Altura } \\
\text { msnm }\end{array}$ & $\begin{array}{c}\text { Temperatura } \\
\text { media } \\
\text { anual, }{ }^{\circ} \mathrm{C} \\
\end{array}$ \\
\hline $\begin{array}{c}\text { Villa } \\
\text { Mañihuales }\end{array}$ & $1986-2004$ & 451003 & 720900 & 150 & 9.38 \\
\hline $\begin{array}{c}\text { Coyhaique } \\
\text { Escuela } \\
\text { Agrícola }\end{array}$ & 198 & 453 & 720200 & 43 & 8.63 \\
\hline Villa Ortega & $1980-2004$ & 452203 & 715901 & 550 & 7.60 \\
\hline Balmaceda & $1980-2004$ & 455500 & 714100 & 520 & 6.97 \\
\hline Ñirehuao & 1993-2004 & 451503 & 714301 & 535 & 8.18 \\
\hline $\begin{array}{c}\text { Coyhaique } \\
\text { Alto } \\
\end{array}$ & $1962-2004$ & 452903 & 713601 & 730 & 7.31 \\
\hline
\end{tabular}

La definición de almacenamiento saturado y no saturado permite la subjetividad de definir cual es el límite físico entre ambos, ya que sobre el nivel freático existe capilaridad que permite que ascienda agua, además la cantidad de agua que puede retener un suelo por capilaridad oscila desde 5\% para suelos muy arenosos hasta un 35\% en suelos arcillosos (Teuscher et al., 1985). Además, el nivel freático oscila a nivel mensual lo que permite que aumente o disminuya el volumen potencial de almacenamiento del subsuelo. Todo lo anterior dificulta la cuantificación de estos almacenamientos en forma local y más aún de la realidad global de la cuenca.

El almacenamiento en lagos debe ser determinado en forma indirecta, ya que no existen mediciones de niveles en los lagos de la cuenca, sin embargo, la magnitud de área ocupada por estos no supera el $0.7 \%$, por lo que su participación en el balance es mínima. El almacenamiento en los cauces de los ríos, tampoco puede ser determinado directamente, por lo que se agrupará con el almacenamiento de los lagos en un almacenamiento superficial. Según información oficial en la cuenca no existen glaciares de importancia, por lo que su almacenamiento se estima de muy baja magnitud. Finalmente el almacenamiento en los mantos de nieve es muy importante, por lo que es necesario concentrar esfuerzos en estimar adecuadamente este aporte, para lo cual se utiliza el concepto de área nival en base a la curva hipsométrica y la temperatura umbral a la que la precipitación líquida se convierte en sólida, la que se estima en forma indirecta.

\section{Modelo parsimonioso}

Un modelo parsimonioso está basado en una simplificación de la representación de los procesos hidrológicos que ocurren en una cuenca de tal forma que sólo sea necesario calibrar un número reducido de parámetros (Xu y Singh, 1998, Orellana et al., 2008). Este enfoque de modelación surgió por la necesidad de obtener información hidrológica relevante para la gestión de los recursos hídricos de cuencas que cuentan con información estadística limitada y se utiliza para análisis de balance hídrico a escala mensual (Pande et al., 2011; Coutu et al., 2012).

El modelo que se utilizó para este trabajo fue desarrollado por el Departamento de Ingeniería Civil y Ambiental de la Universidad de Tufts en Medford, Estados Unidos (Limbrunner et al., 2005) y se basa en el uso del método de la curva número para evaluar la infiltración y generación de escorrentía superficial a paso diario, realizando el balance posteriormente a paso mensual. El modelo original propuesto por Limbrunner et al. (2005) considera cuatro parámetros para simular la evapotranspiración, la variación de la humedad del suelo, almacenamiento en un acuífero saturado con la generación de flujo base, acumulación y derretimiento de nieve y almacenamiento en los cauces superficiales. Sin embargo, el modelo resultó ser muy simplista para representar los procesos hidrológicos de la cuenca del Simpson, por lo que fue necesario considerar dos parámetros adicionales $\left(\mathrm{T}_{\mathrm{b}} \mathrm{y} \alpha\right)$, que permitieron mejorar la predictibilidad del modelo.

\section{Resumen del modelo}

La esencia del modelo podría resumirse en que el caudal en el punto de cierre de la cuenca es la suma de la escorrentía directa desde la precipitación, el derretimiento y el flujo base. La evapotranspiración remueve agua desde la cuenca, la cual sale del agua almacenada en el subsuelo no saturado. Las ecuaciones principales que gobiernan el modelo se presentan a continuación. La primera de ellas, la infiltración I $(\mathrm{t})$, se definió como la diferencia entre la precipitación efectiva $\mathrm{P}_{\mathrm{e}}(\mathrm{t})$ y la escorrentía directa $\mathrm{R}(\mathrm{t})$ que ingresa al suelo no saturado.

$I(t)=P_{e}(t) R(t)$

La precipitación efectiva es la suma de la lluvia caída y el derretimiento. La ecuación de continuidad en el suelo no saturado está dada en función de una expresión recursiva 
del almacenamiento previo $\mathrm{S}(\mathrm{t}-1)$, infiltración $\mathrm{I}(\mathrm{t})$, percolación Perc(t) y evapotranspiración ET(t).

$$
\mathrm{S}(\mathrm{t})=\mathrm{S}(\mathrm{t}-1)+\mathrm{I}(\mathrm{t})-\operatorname{Perc}(\mathrm{t})-\mathrm{ET}(\mathrm{t})
$$

El almacenamiento de la zona de agua subterránea $\mathrm{G}(\mathrm{t})$ es una función del almacenamiento previo, del flujo base $\mathrm{k}_{\mathrm{g}} \mathrm{G}(\mathrm{t}-1)$ y la percolación.

$$
\mathrm{G}(\mathrm{t})=\mathrm{G}(\mathrm{t} 1)+\operatorname{Perc}(\mathrm{t}) \mathrm{k}_{\mathrm{g}} \mathrm{G}(\mathrm{t} \quad 1)
$$

El caudal a la salida de la cuenca entregado por la red de drenaje, la cual transporta el flujo base y la escorrentía, es calculado según:

$$
\mathrm{Q}(\mathrm{t})=\mathrm{kn}\left[\mathrm{N}(\mathrm{t}-1)+\mathrm{R}(\mathrm{t})+\mathrm{k}_{\mathrm{g}} \mathrm{G}(\mathrm{t}-1)\right]
$$

donde $\mathrm{kn}$ es un parámetro de escorrentía pluvial adimensional. El almacenamiento superficial en la red de drenaje tiene un almacenamiento igual a $\mathrm{N}(\mathrm{t})$ donde:

$$
N(t)=(1-k n)\left[N(t-1)+R(t)+k_{g} G(t-1)\right]
$$

La primera modificación realizada a este modelo es considerar la superficie nival para dividir la precipitación media de la cuenca $\mathrm{P}(\mathrm{t})$, se considera que existe precipitación líquida solo fuera de esta superficie y por lo tanto la precipitación efectiva se transforma en:

$$
P_{e}(t)=P(t)\left(1-\frac{A_{\text {nival }}}{A_{\text {cuenca }}}\right)+P_{S}(t), \text { si } T>0
$$

En el caso que la temperatura sea menor que cero, entonces no existe derretimiento $P_{s}(t)$ y se mantiene la ponderación de áreas. El área nival se obtiene en base a la curva hipsométrica de la cuenca y la altura de la isoterma de congelación o precipitación sólida. La máxima humedad permitida en la zona no saturada, se calcula con el método de curva número, reemplazando por el menor valor posible de curva número $\mathrm{CN}_{1}$.

$$
\mathrm{S}_{\max }=\frac{25400}{\mathrm{CN}_{1}}-254
$$

Sin embargo, se realizó una modificación a la ecuación (13), ya que, la percolación no se produce solo cuando la humedad del suelo sobrepasa este valor, sino a un valor menor debido al movimiento descendente del agua producto de la diferencia de energía. Con lo cual, (13) se ajusta por un coeficiente $\alpha \leq 1$, que corresponde a otro de los parámetros del modelo.

$$
\mathrm{S}_{\max }=\alpha\left(\frac{25400}{\mathrm{CN}_{1}}-254\right)
$$

Además, se modifica el almacenamiento nival considerando su superficie. Las ecuaciones son:

$$
\operatorname{Snow}(t)=\operatorname{Snow}(t-1)+P(t) \frac{A_{\text {nival }}(T)}{A_{\text {cuenca }}}-P_{S}
$$

$\hat{P}_{S}(t)=\left\{\begin{array}{cc}M\left(T-T_{b}\right), & T>T_{b} \\ 0, & T \leq T_{b}\end{array}\right.$

donde $\mathrm{M}$ es un parámetro de derretimiento de nieve en $\mathrm{mm} /{ }^{\circ} \mathrm{C}$.

$P_{S}(t)=\left\{\begin{array}{l}\hat{P}_{S}(t), \operatorname{Snow}(t-1)+P(t) \frac{A_{\text {nival }}(T)}{A_{\text {cuenca }}}-\hat{P}_{S}>0 \\ \text { Snow }(t-1)+P(t) \frac{A_{\text {nival }}(T)}{A_{\text {cuenca }}}, \text { en otro caso }\end{array}\right.$

Donde $\hat{\mathrm{P}}_{\mathrm{S}}$ es el derretimiento potencial y $\mathrm{T}_{\mathrm{b}}$ es la temperatura base del método grado-día. La percolación es calculada primero obteniendo la humedad total que debiera almacenarse si no existiera otro almacenamiento subterráneo, $\mathrm{SS}(\mathrm{t})=\mathrm{S}(\mathrm{t})+\mathrm{I}(\mathrm{t})$, y luego en base a (18).

$\operatorname{Perc}(\mathrm{t})=\left\{\begin{array}{cc}\mathrm{SS}(\mathrm{t})-\mathrm{S}_{\max }+\mathrm{k}_{\mathrm{S}} \mathrm{S}(\mathrm{t}-1), & \text { si } \mathrm{SS}(\mathrm{t})>\mathrm{S}_{\max } \\ \mathrm{k}_{\mathrm{S}} \mathrm{S}(\mathrm{t}-1) & \text {, en otro caso }\end{array}\right.$

Donde $\mathrm{k}_{\mathrm{s}}$ es el coeficiente que permite que exista percolación durante todos los meses a pesar que no se supere el $\mathrm{S}_{\max }$.

\section{Resultados}

para la generación de la precipitación en la cuencas se utilizan patrones de distribución espacial de precipitación media mensual estimados previamente a través de métodos geoestadísticos (Co-krigging). La serie de tiempo de precipitación en toda la cuenca se realiza considerando 
una proporción directa con la estación base, es decir, se analiza la proporción que existe entre el patrón de cada mes y el promedio mensual de la estación base, y se aplica este mismo coeficiente para amplificar o disminuir el valor medio de la cuenca según corresponda.

La evapotranspiración potencial estimada en base a la ecuación de Hargreaves y Samani (1985) se consideró constante entre años. Sin embargo, frente a la posible variación que se produce por la desviación que se presenta entre el mismo mes de distintos años, se analizó la variación que se presenta al considerar la desviación estándar de las temperaturas y se concluyó que dado que se trabaja con valores medios mensuales, esta desviación no era importante y no afectaba sustancialmente la evapotranspiración potencial entre años.

El valor de curva número promedio de la cuenca se obtiene considerando información de textura de suelo en conjunto con el uso de suelo disponible, dando como resultado $\mathrm{CN}=$ 60.5. La temperatura media de la cuenca para toda la serie de tiempo, se obtiene realizando una comparación, para todos los meses, entre la estación base y la temperatura media mensual de la estación. La diferencia que se produce entre éstas, es asumida como constante para toda la cuenca, al igual que en la precipitación areal.

\section{Modelación}

El resultado de los parámetros se ha obtenido de un proceso de calibración de 10 años y los siguientes 10 años se utilizaron para realizar la validación (Figuras 3 y 4). Los valores medios de un ciclo anual se muestran en la Figura 5.

Las condiciones iniciales se consideraron como el promedio de cada almacenamiento del mes de marzo, por esta razón, estos son presentados como resultados (Tabla 5).

Tabla 5: Resumen de parámetros y condiciones iniciales.

\begin{tabular}{|c|c|c|c|}
\hline \multicolumn{2}{|c|}{ Parámetros } & \multicolumn{2}{|c|}{ Condiciones iniciales, $\mathrm{mm}$} \\
\hline $\mathrm{k}_{\mathrm{g}}$ & 1.056 & S inicial & 138 \\
\hline $\mathrm{kn}$ & 0.527 & $\mathrm{G}$ inicial & 4 \\
\hline $\mathrm{k}_{\mathrm{S}}$ & 0.016 & $\mathrm{~N}$ inicial & 11 \\
\hline $\mathrm{M}$ & 5.861 & Snow inicial & 0 \\
\hline $\mathrm{T}_{\mathrm{b}}$ & 0.355 & & \\
\hline$\alpha$ & 0.513 & & \\
\hline
\end{tabular}

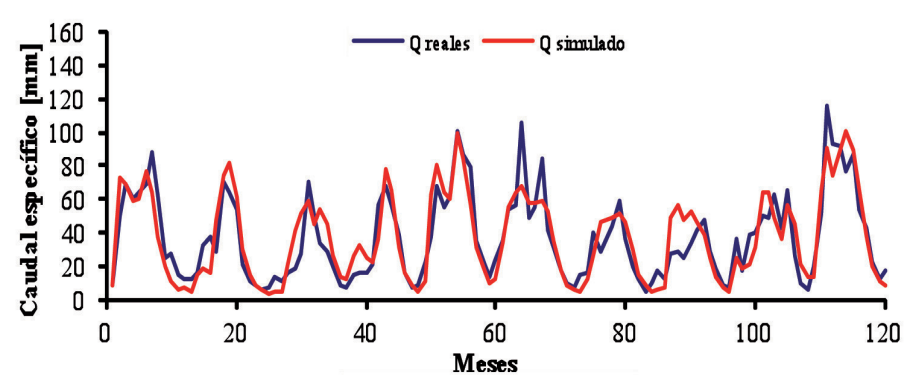

Figura 3: Etapa de calibración del modelo

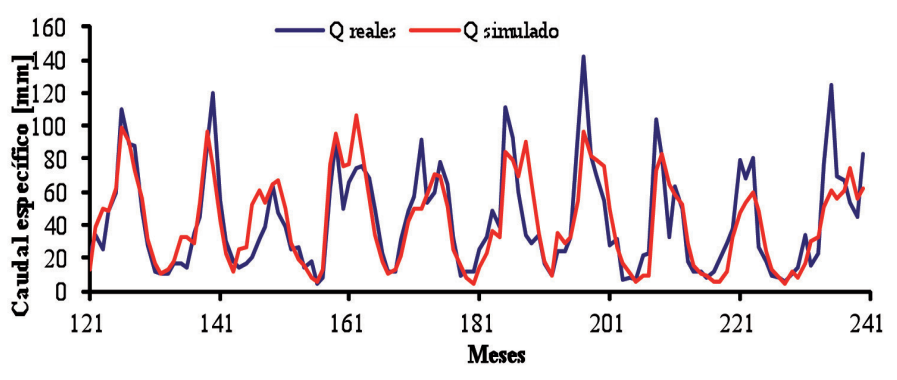

Figura 4: Etapa de validación del modelo

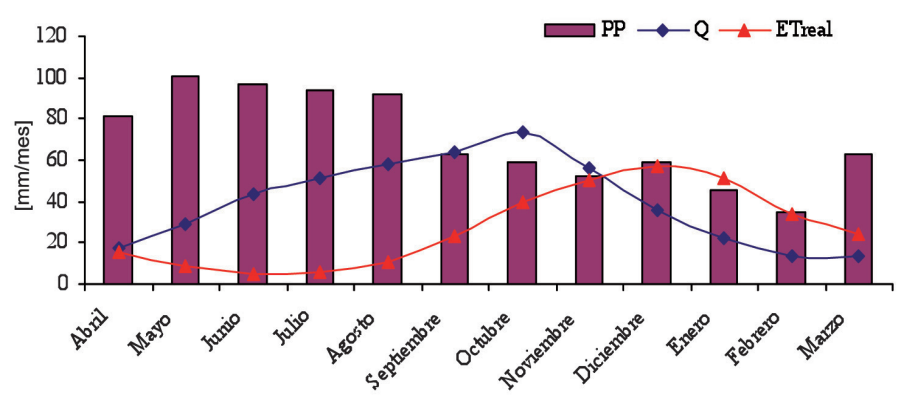

Figura 5: Ciclo medio anual de las entradas y salidas a la cuenca

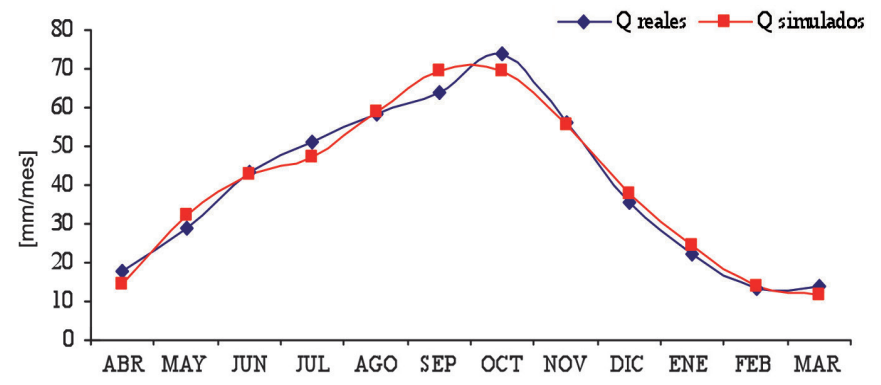

Figura 6: Comparación entre caudales medios mensuales reales y simulados 
Tabla 6: Balance Hídrico Anual simplificado desde 1961 hasta 2001

\begin{tabular}{|c|c|c|c|c|c|c|}
\hline Periodo & $\begin{array}{c}\text { Precipitaciones } \\
\mathrm{mm}\end{array}$ & $\begin{array}{c}\text { Escorrentía } \\
\mathrm{mm}\end{array}$ & $\begin{array}{c}\text { Evapotranspiración } \\
\text { Real } \mathrm{mm}\end{array}$ & $\begin{array}{c}\text { Evaporación } \\
\mathrm{mm}\end{array}$ & $\begin{array}{c}\text { PP-Q-ETR } \\
\mathrm{mm}\end{array}$ & Error \% \\
\hline $1962 / 1981$ & 1458 & 884 & 440 & 2,57 & 131.43 & $-9.0 \%$ \\
\hline $1982 / 2001$ & 839.3 & 478.6 & 325.2 & - & 35.5 & $-4.2 \%$ \\
\hline
\end{tabular}

Tabla 7: Balance Hídrico Anual completo desde 1981/1982 hasta 2000/2001

\begin{tabular}{|c|c|c|c|c|c|c|c|c|c|c|}
\hline $\begin{array}{c}\text { Precip. } \\
\mathrm{mm}\end{array}$ & $\begin{array}{c}\text { Escorr. } \\
\mathrm{mm}\end{array}$ & $\begin{array}{c}\text { ETR } \\
\mathrm{mm}\end{array}$ & $\begin{array}{c}\text { PP } \\
\text {-Q-ETR }\end{array}$ & $\begin{array}{c}\text { Sub sup. } \\
\mathrm{mm}\end{array}$ & $\begin{array}{c}\text { Superf. } \\
\mathrm{mm}\end{array}$ & $\begin{array}{c}\text { Subterr. } \\
\mathrm{mm}\end{array}$ & $\begin{array}{c}\text { Nieve } \\
\mathrm{mm}\end{array}$ & $\begin{array}{c}\Delta \mathrm{S}+\Delta \mathrm{N}+ \\
\Delta \mathrm{G}+\Delta \mathrm{Nieve}\end{array}$ & $\begin{array}{c}\text { error } \\
\mathrm{mm}\end{array}$ & $\begin{array}{c}\text { error } \\
\%\end{array}$ \\
\hline 839.30 & 478.62 & 325.21 & 35.47 & 0.88 & 2.40 & 1.81 & 0.00 & 5.09 & -30.38 & -3.62 \\
\hline
\end{tabular}

Tabla 8: Resumen del Balance Hídrico Mensual promedio 1981/1982 hasta 2000/2001

\begin{tabular}{|l|c|c|c|c|c|c|c|c|c|}
\hline \multicolumn{1}{|c|}{ Mes } & $\begin{array}{c}\mathrm{PP} \\
\mathrm{mm}\end{array}$ & $\begin{array}{c}\mathrm{Q} \\
\mathrm{mm}\end{array}$ & $\begin{array}{c}\mathrm{ETR} \\
\mathrm{mm}\end{array}$ & $\begin{array}{c}\mathrm{S} \\
\mathrm{mm}\end{array}$ & $\begin{array}{c}\mathrm{N} \\
\mathrm{mm}\end{array}$ & $\begin{array}{c}\mathrm{G} \\
\mathrm{mm}\end{array}$ & $\begin{array}{c}\text { Nieve } \\
\mathrm{mm}\end{array}$ & $\begin{array}{c}\text { error } \\
\mathrm{mm}\end{array}$ & $\begin{array}{c}\text { error } \\
\%\end{array}$ \\
\hline Abril & 81.00 & 17.58 & 15.30 & 175.40 & 12.76 & 7.85 & 0.00 & 1.19 & 1.47 \\
\hline Mayo & 100.40 & 29.10 & 8.95 & 195.22 & 28.92 & 28.32 & 0.00 & -5.90 & -5.88 \\
\hline Junio & 96.60 & 43.23 & 5.21 & 199.59 & 38.26 & 33.69 & 26.57 & -2.49 & -2.58 \\
\hline Julio & 94.00 & 50.95 & 5.59 & 199.08 & 42.36 & 28.75 & 65.95 & 0.58 & 0.61 \\
\hline Agosto & 91.50 & 58.26 & 11.11 & 193.58 & 52.75 & 44.23 & 64.05 & -3.68 & -4.02 \\
\hline Septiembre & 63.30 & 63.69 & 23.62 & 181.24 & 62.31 & 37.32 & 40.92 & -8.80 & -13.91 \\
\hline Octubre & 59.00 & 74.02 & 39.59 & 165.66 & 62.09 & 29.57 & 11.83 & 1.98 & 3.35 \\
\hline Noviembre & 51.80 & 56.24 & 50.00 & 152.10 & 50.04 & 8.89 & 1.55 & -2.12 & -4.10 \\
\hline Diciembre & 58.60 & 35.79 & 56.74 & 133.79 & 33.97 & 6.43 & 0.00 & -4.47 & -7.63 \\
\hline Enero & 45.70 & 22.45 & 50.81 & 117.81 & 21.77 & 3.11 & 0.00 & -3.93 & -8.59 \\
\hline Febrero & 34.90 & 13.56 & 33.95 & 113.38 & 12.63 & 1.70 & 0.00 & -2.38 & -6.83 \\
\hline Marzo & 62.50 & 13.75 & 24.32 & 138.24 & 10.58 & 3.48 & 0.00 & 0.16 & 0.26 \\
\hline
\end{tabular}

\section{Balance hídrico}

Una vez aplicado el modelo es posible obtener el balance hídrico anual y representar el promedio mensual de las variables del balance, que se muestra en las Tablas 6 y 7. Ahora, realizando el balance hídrico anual completo, que considera la variación de los almacenamientos, se obtiene un aporte de $5.09 \mathrm{~mm}$ de estos, lo cual valida el supuesto tradicional de considerarlos despreciables.

El resumen del balance hídrico mensual se encuentra en la Tabla 8, donde se han utilizado las siguientes abreviaciones: precipitación PP, escorrentía Q, evapotranspiración real ETR, almacenamiento subsuperficial o no saturado $\mathrm{S}$, almacenamiento superficial $\mathrm{N}$, almacenamiento subterráneo o saturado G y almacenamiento nival (Nieve). En este balance se presentan los valores promedios mensuales de cada variable y el error promedio mensual del balance de los 240 meses considerados.

\section{Discusión}

La calidad de la información utilizada y la forma en como se utilizan los datos, puede de una u otra forma afectar los resultados de la modelación. Por esta razón es importante tener presente las limitaciones o simplificaciones utilizadas. En este caso las principales se encuentran asociadas al punto de vista simplificado en el cual la precipitación y la temperatura se expandieron hacia la cuenca. El uso de estaciones patrones para extrapolar la realidad de la cuenca a través de planos de isoyetas e isotermas medias puede no ser siempre muy representativo. En el caso de la precipitación puede alejarse considerablemente para eventos extremos, y para las isotermas el efecto del viento puede trasladar masas de aire afectando al gradiente térmico y por ende a la evapotranspiración. Sin embargo, la escasez de datos en una zona tan extensa (solo 4 estaciones pluviométricas y 3 fluviométricas dentro de una cuenca 
de $3972 \mathrm{~km}^{2}$ ) y la extensión de periodo de modelación impiden una mejor estimación.

$\mathrm{Al}$ analizar los caudales simulados y reales en las Figuras $3,4,5$ y 6 es posible observar que el modelo no predice completamente los caudales máximos, lo cual puede ser atribuido a la existencia de eventos de precipitación que se escapan de los supuestos planteados. En general muchos fenómenos pueden inducir este tipo de errores o simplemente puede ser asumido directamente a que el modelo no es capaz de capturar todos los fenómenos que se encuentran integrados en una cuenca, de cualquier modo los estadígrafos obtenidos en la calibración se consideraron adecuados (coeficiente de correlación de 0.88 y error cuadrático medio de $1.13 \mathrm{~mm}$ ) y en la etapa de validación (coeficiente de correlación de 0.83 y error cuadrático medio de $1.56 \mathrm{~mm}$ ). Al realizar un análisis de residuales se obtiene una media de $-0.11 \mathrm{~mm}$, coeficiente de asimetría de -0.34 y una curtósis de 2.20 , valores que son muy cercanos a una distribución normal por lo que el modelo se considera satisfactorio.

Al realizar la comparación entre el balance hídrico anual desde 1981/1982 hasta 2000/2001 y el balance anterior desde 1961/1980, se aprecia una gran diferencia en cuanto a la precipitación media areal y escorrentía. En el caso de la precipitación, al realizar una comparación entre la información utilizada en el balance anterior para las 3 estaciones insertas en la cuenca, se tiene una disminución de $-14 \%$ en Coyhaique Alto, en Balmaceda en un -33\% y en Coyhaique Escuela Agrícola en un -21\%, a su vez al analizar la escorrentía el valor promedio anual actual es de $476.9 \mathrm{~mm} /$ año en cambio el valor anterior corresponde a $884 \mathrm{~mm} / a$ ño. Sin embargo se encontró que la estimación del área de la cuenca en el balance anterior se encuentra subestimada en un $8 \%$, lo cual aumentaría su escorrentía específica, no obstante este error no soluciona substancialmente la discrepancia entre ambas escorrentías. Más aún, al comparar los caudales medios mensuales en la estación de Coyhaique bajo junta Coyhaique (Figura 7), se aprecia que existe una clara disminución de los caudales entre ambos balances.

Ahora, si se realiza una comparación entre el balance hídrico simplificado y el completo para nuestro periodo de estudio, es posible observar que el supuesto de considerar la variación de los almacenamientos igual a cero, es totalmente factible y adecuado en el caso de ser necesario, ya que la discrepancia entre ambos errores es aproximadamente $5 \mathrm{~mm}$. En cuanto a la disponibilidad de agua es posible mencionar que la cuenca presenta 4 meses con déficit hídrico y 6 meses con exceso de agua. Además, es de carácter subhúmedo, ya que el período del año en que el suministro de humedad aprovechable del suelo alimentado por la lluvia es mayor que la mitad de la evapotranspiración de referencia, corresponde a 240 días (CAZALAC, 2004).

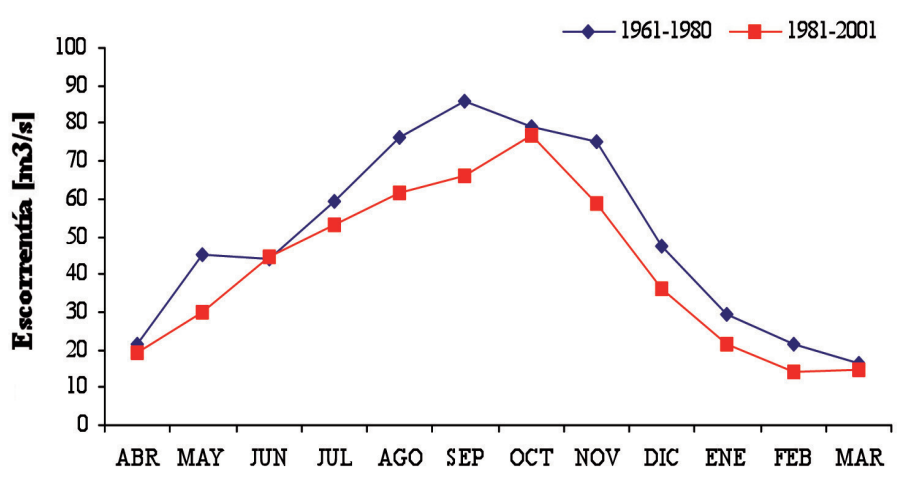

Figura 7: Comparación de escorrentía entre balance hídrico para la estación de Simpson bajo junta Coyhaique

\section{Conclusiones}

Del balance hídrico, se tiene que la precipitación media anual es de $839.3 \mathrm{~mm} / a n ̃ o$, la escorrentía es de $478.6 \mathrm{~mm} /$ año y la evapotranspiración real es de $325.2 \mathrm{~mm} / \mathrm{año}$, lo cual arroja un error de cierre de $-35.5 \mathrm{~mm} / \mathrm{año}$, en el caso del balance anterior (1961-1980) el error de cierre fue de $131.4 \mathrm{~mm} / a n ̃ o$. De aquí se observa que la utilización de metodologías más modernas mejora considerablemente el error de cierre, casi $100 \mathrm{~mm} / \mathrm{año}$ de diferencia y una disminución a la mitad del error porcentual, además de tener la facilidad de determinar cada una de las variables en forma independiente lo que minimiza la trasmisión de errores entre variables. La disponibilidad de agua en la cuenca del río Simpson es de $40.648 \mathrm{~m}^{3}$ /año/por persona, lo cual sitúa la cuenca a la altura de valores de la región de la Araucanía y muy por debajo de los valores medios de la región de Aysén, $3.362 .822 \mathrm{~m}$ 3/año/por persona, lo cual hace de la cuenca, a pesar de la diferencia, muy propensa a desarrollarse sin limitaciones hídricas. 
Al comparar las magnitudes de los componentes del balance actual y anterior, se encontró una disminución de $42.4 \%$ en la precipitación y de $45.9 \%$ en la escorrentía, lo que podría ser asumida como error en la estimación de alguno de los balances. Sin embargo, si se analiza las productividades hídricas por unidad de precipitación, se tiene en el balance anterior $0.61 \mathrm{~mm} / \mathrm{mm}$ y en el actual 0.57 $\mathrm{mm} / \mathrm{mm}$, lo cual desecha la posibilidad de un error grosero en algunos de los balance. Por otro lado, al analizar la metodología empleada en el balance realizado por la DGA (1987), se tiene que probablemente las isoyetas creadas sobrestimen la precipitación producto de la escasez de estaciones en el periodo en estudio y la no consideración del marcado gradiente de precipitaciones de Oeste a Este, lo cual permitiría que las variaciones entre los balances no fuesen tan marcadas. En el futuro, con el fin de eliminar posibles diferencias, se debiera realizar en forma periódica estos balances y a través de metodologías estandarizadas, ya que con solo dos balances realizados y metodologías muy distintas es difícil determinar fehacientemente si es que existen problemas en la determinación del balance o existen cambios en el comportamiento de la cuenca en estudio. En conclusión sin esta modelación, variables como evapotranspiración real no hubiesen sido posibles de obtener sin la necesidad de utilizar fórmulas empíricas, determinar características de la sensibilidad de la cuenca ante cambios naturales o producto del hombre, hubiesen estado muy lejanas de realizar, por lo tanto, todos los resultados expuestos en este trabajo reafirman la utilidad de la modelación para permitir abordar realidades complejas de cualquier fenómeno y manipularlo para estudiar su comportamiento.

\section{Agradecimientos}

Se agradece al Proyecto Fondecyt No 1050576 "Efectos geológicos y ambientales de la dinámica geomorfológico e hidrológica de la hoya del río Aysén" y también la Dirección General de Aguas, DGA Chile, por proporcionar la información con que se desarrolló la investigación.

\section{Referencias}

CAZALAC (2004). Guía Metodológica para la elaboración del mapa de zonas áridas, semiáridas y subhúmedas secas de América Latina y el Caribe. Centro del Agua para Zonas Áridas y Semiáridas de América Latina y El Caribe (CAZALAC), 66p. Coutu, S., Giudice, D.D., Rossi, L. and Barry, D.A. (2012). Parsimonious hydrological modeling of urban sewer and river catchments. Journal of Hydrology 464-465, 477-484.

DGA (1987). Balance Hídrico de Chile. Dirección General de Aguas. Ministerio de Obras Públicas. Chile.

DGA (2004). Cuenca del Río Aysén. Diagnóstico y clasificación de los cursos y cuerpos de agua según objetivos de calidad. Dirección General de Aguas. Ministerio de Obras Públicas. Chile. Realizado por CADE-IDEPE.

Hargreaves, G.H. and Samani, Z.A. (1985). Reference crop evapotranspiration from temperature. Applied Engineering in Agriculture 1(2), 96-99

Limbrunner, J.R., Vogel, R.M. and Chapra, S. (2005). A Parsiminous Watershed Model. In Watershed Models, Singh, V.P. and Frevert, D.K editors, CRC Press, 549-567.

Orellana, B., Pechlivanidis, I.G., McIntyre, N., Wheater, H.S. and Wagener, T. (2008). A Toolbox for the Identification of Parsimonious Semi-Distributed Rainfall-Runoff Models: Application to the Upper Lee Catchment. International Congress on Environmental Modelling and Software Integrating Sciences and Information Technology for Environmental Assessment and Decision Making, Barcelona, Spain.

Pande S., Savenije, H. G., Bastidas, H. G. and Gosain, A.K. (2011). A Parsimonious Hydrological Model for a Data Scarce Dryland Region. Water Resource Management, 26(4), 909-926

Sokolov, A. y Chapman, T. (1981). Métodos de Cálculo del Balance Hídrico. Guía Internacional de investigación y métodos. Instituto de Hidrología de España - UNESCO

Teuscher, H., Adler, R. y Seaton, J. (1985). El suelo y su fertilidad. Editorial Continental. México.

UNESCO-IMTA (2006). Evaluación de Recurso Hídricos. Elaboración de Balances Hídricos por cuencas hidrográficas. Instituto Mexicano de Tecnología del Agua. México.

Xu, C.Y. and Singh, V.P. (1998). A Review on Monthly Water Balance Models for Water Resources Investigations. Water Resources Management 12, 31-50. 